\title{
USING MULTI-AGENT TECHNOLOGY FOR THE DISTRIBUTED MANAGEMENT OF A CLUSTER OF REMOTE SENSING SATELLITES
}

\author{
P. SKOBELEV ${ }^{1}$, E. SIMONOVA ${ }^{1,2}$ \& A. ZHILYAEV 3 \\ ${ }^{1}$ Smart Solutions, Ltd, Russia. \\ ${ }^{2}$ Department of Informatics, Samara State Aerospace University, Russia. \\ ${ }^{3}$ Department of Aircraft Engineering, \\ Samara State Aerospace University, Russia.
}

\begin{abstract}
In recent years, the interest for space missions involving multiple satellites has been rapidly growing. This trend is responsible for an increasing need for management strategies able to coordinate the different satellites and to allocate tasks among them. In most existing systems, the management of a cluster of satellites is organized centrally and statically, which significantly reduces the responsiveness to changes in the target environment. Our research aims at designing a distributed dynamic management system where tasks are assigned to a whole constellation of satellites and their performers can adaptively change depending on emerging events. In this paper, we explore the potentiality of multi-agent technology and ontology to develop an intelligent space system. We describe our software prototype and how it can be applied to the planning problem of remote sensing satellites. In this solution, each satellite has been represented as an agent enabling communication, coordination, and negotiation among the other agents to achieve intended business goals. Moreover, we present the results of experiments showing the advantages of the proposed approach.

Keywords: distributed management, multi-agent technology, ontology, remote sensing satellite.
\end{abstract}

\section{INTRODUCTION}

Earth remote sensing (ERS) space systems are designed for objects and processes occurring on the Earth's surface and near space observation. The creation and application of satellite clusters is one of the promising trends in this area [1,2]. Moreover, each spacecraft (SC) can be equipped with various equipments and can have different orbital parameters, and the solution of the sensing problem may require coordinated interaction of several spacecrafts. The management of a cluster of satellites is organized centrally and statically; each satellite has a prearranged order of task performance, set directly from the management center [2]. There is an alternative solution to implementing dynamic control, when the current tasks are given not an individual spacecraft, but to the entire cluster as a whole. This approach will allow us to reallocate tasks between cluster satellites during their implementation.

\section{PROBLEM STATEMENT}

Let there be a swarm of spacecrafts capable of ground objects identification in visible, infrared, and radiation spectrum. Each SC can autonomously identify its space coordinates at any time and has devices for two-way communication with other satellites. Each SC has a cyclogram designing mechanism for its behavior scheduling. This device allows us to determine a mutual visibility with other objects. A user interacts with the swarm of satellites via the network of ground stations, where each one can transmit initial data on tasks to the swarm and receive the performance results.

A satellite cluster is given a task to dynamically identify observation areas with complex properties. In other words, it is about matching the given target object to the data obtained during observation area identification. For that purpose, we need to identify an object as one of the objects in the visible, infrared, or radiation spectrum (a combination of the spectral characteristics is

(C) 2016 WIT Press, www.witpress.com

ISSN: 1755-7437 (paper format), ISSN: 1755-7445 (online), http://www.witpress.com/journals DOI: 10.2495/DNE-V11-N2-127-134 
possible). The observation area has the following attributes: location, priority, and a time slot when the research is needed. The same task can be solved with several satellites.

\section{METHODS}

Using a multi-agent approach is suggested, which has been actively developing in recent years at the junction of parallel systems, distributed problem solving, artificial intelligence, and telecommunications works for dynamic task distribution implementation between spacecraft. Unlike the traditional problem-solving method that searches for a certain deterministic algorithm, allowing us to find the best solution, multi-agent technologies get the solution automatically by the interaction of multiple independent targeted software or hardware modules, that is, agents [3,4].

\subsection{Multi-agent approach to the management of a swarm of spacecrafts}

In multi-agent approach implementation, a remote sensing space system is considered as a heterogeneous multi-agent system, where individual ERS satellites relay satellites that ensure rapid communication between agents in the absence of direct visibility and ground stations represent agents. In this case, a multi-agent system is a global dimensionally distributed network, where agents (nodes) either rotate around the Earth or rotate with it if the node is situated on the surface [5]. Space system management is implemented by a coordinated interaction of the network nodes. In this interaction, satellites dynamically form a team, distribute the given task between them, and solve it by parts, considering their location and on-board equipment abilities (Fig. 1).

1. At any moment in time, the ground station (represented by a 'radar' sign) specifies and transmits the task of a target object (represented by a 'home' sign) to search all the visible satellites (1). The tasks can be unknown to the satellites beforehand and require the cooperation of several satellites. The observation area is divided into blocks.

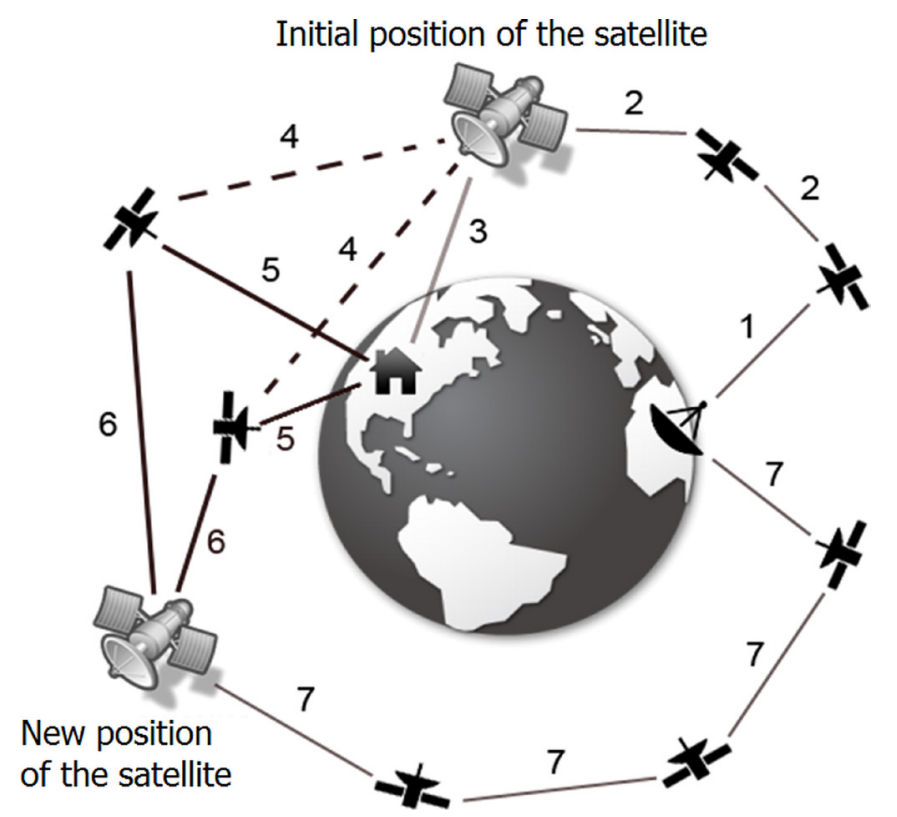

Figure 1: Network agents' interactions scheme. 
2. The satellites move in orbits with different characteristics. If the satellite does not 'see' the observation area, it operates as a re-translator, sending signals to the closest visible satellites. Since the satellite that received a request is far from the observation area, it transmits the request to the satellite closest to the observation area, which is in its radio availability area. The process of request transmitting continues until it reaches a satellite above the observation area (2). Among the several appropriate satellites that can identify the target, we choose the one that will fly over the observation area before the others or the one that stays in the visibility of an observation area longer, which is determined in the negotiations of the satellite team that can see the observation area.

3. If the satellite that receives the task has the required identification equipment, it sends a message to other satellites, letting them know that it will take the task. While the satellite moves in an orbit above the observation area, it performs identification, searching for the target object in its spectrum. If the satellite does not succeed in its search and leaves the visibility area, it transmits the request to the other satellite that is entering the observation area. The process continues until a satellite identifies the object in its wave range that fits the description provided by the ground station. Let us call this satellite an initiator (3).

4. Simultaneously, the satellite-initiator transmits the request of area research to other satellites operating in a different wavelength range (4).

5. Satellites of these ranges check the specified object by the coordinates transmitted by the satellite-initiator (5).

6. Satellites send the research results to the satellite-initiator directly or through the re-translators network (6).

7. If the research result is positive, then the satellite-initiator sends a message with it to the ground station. After that, the satellite-initiator continues the search for other objects that fit the description given by the ground station in the observation area (7).

8. The scanning is finished with the ground station signal or when all of the observation area blocks are searched by the equipped satellites that work in all the wavelength ranges.

A multi-agent approach application allows us to adaptively reallocate the tasks inside the swarm by the interaction between the spacecrafts. Moreover, in this type of system, it becomes possible to process the unscheduled events, such as new top-priority task arrival, spacecraft failure, placing new spacecraft into the orbit, etc. Spacecrafts can not only independently and individually make decisions, but also negotiate and flexibly form satellite alliances (or teams) of different purpose if necessary. At the same time, the signal that initiates rescheduling of the whole swarm can be sent both from the Earth's surface and from any of the satellites, for example, from the one that was first to discover the target object.

\subsection{Ontological description of the target object}

The aim of an ERS spacecraft swarm is to establish a match between the map of the observation area and a specified pattern that describes the ratio of certain basic elements such as a house or a road between them. An ontology implemented as a semantic network is used for a target object description formalization. An ontology is a conceptual domain knowledge represented in a computerreadable format and applied in decision-making. Ontology conceptual knowledge means that the knowledge is formulated in terms of basic concepts (the most common entities and relations) that describe domain area fragments [6]. A semantic network is a directed graph, where nodes represent some concepts and the arcs are the relations between them.

A target object description is sent from the ground station in the form of a tree of connections, where the terminal nodes (leaves) are basic concepts, nonterminal nodes are composite concepts, 


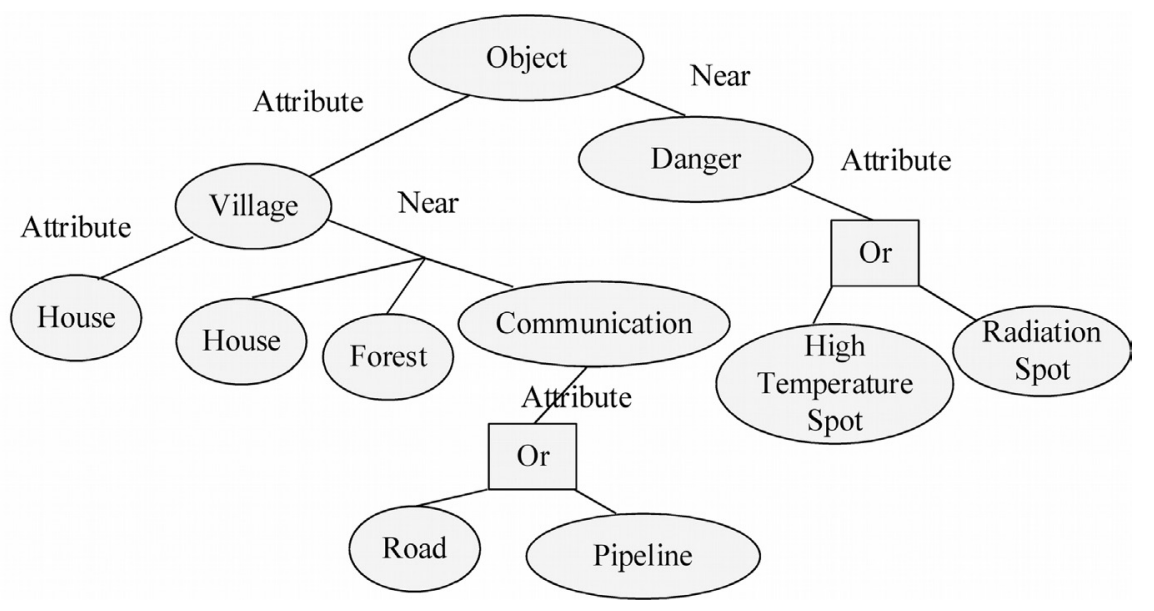

Figure 2: Ontological description of a target object.

and the arcs are the connections between them. Generally, the tree can contain concepts of different types of spectrum. A composite concept can be made of the basic ones with the relations 'not', 'or', 'center', 'near', 'before', etc. An example of an ontological description of a target object in a tree form is shown in Fig. 2.

According to the description in Fig. 2, the target object is a house in a village that has the additional attributes of high temperature or radiation level. The 'village' concept is defined as a group of houses with a forest, a road, and a pipeline nearby.

A spacecraft that received a request for the observation area sensing extracts a subtree from the whole tree, containing only those concepts that are in its visibility area. The spacecraft performs a sensing according to its ontological description subtree and in the case of a positive outcome, sends a request to spacecraft for sensing in other spectra, transmitting a corresponding subtree along with the task description.

A spacecraft of a corresponding visibility spectrum can explore each subtree of the ontological description of a target object regardless of the spacecraft of the other spectra, except when the different spectra concepts are connected by the 'or' or 'not' relations. For example, if an SC of a radiation spectrum explored the observation area and did not find a high radiation level, then it cannot make a conclusion that the observation area does not fit the ontological description, because it may contain a high temperature object.

Ontologies application allows us to formalize specific domain knowledge in a computer-readable format and separate this knowledge from the system software code. This creates a basis for further system development and the addition of new features without constant reprogramming.

\section{RESULTS}

\subsection{Simulation system}

A simulation system for distributed management research methods of moving objects in the satellite clusters was developed (Fig. 3). An ontology creation module and dynamic scheduling module that form a processing plan of the flow of incoming events (observation area sensing request, equipment 


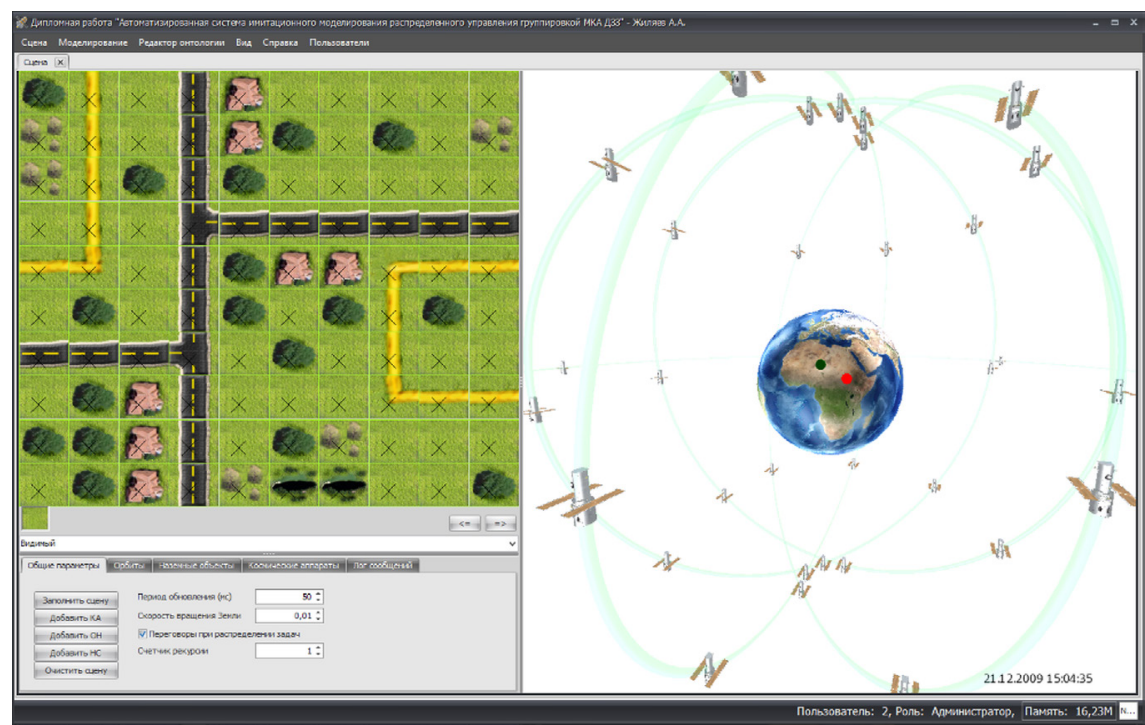

Figure 3: Simulation system screen.

failure, etc.) and perform adaptive dynamic change of the created plan are the main components of the simulation system.

\subsection{Experimental research}

Let us use the following satellites cluster model. Let there be 32 spacecrafts with different spectrum equipments and three relay satellites that are situated on the geostationary orbit in the ERS. The task is the sensing of several closely situated observation areas, whose number in different series of experiments can change from one to six.

Two groups of 30 tests are carried out during the experiment:

1. new tasks are centrally allocated to the most appropriate SC without considering their current loading (batch scheduling) and

2. rescheduling is performed when a new order arrives, by the means of interaction (negotiations) between the satellites (real-time scheduling).

Moreover, numerous tests are divided into six series that differ in the number of observation areas in the scene.

Table 1 shows the time values (in terms of simulated time) spent on the observation areas' exploration and that are obtained during the experiment. The values of arithmetic mean and standard deviation of the exploration time of the observation areas are shown for each series of tests.

A graphical representation of the experimental results is shown in Fig. 4.

Using agents' negotiations allows us to significantly decrease the standard deviation of the amount of time spent on the observation areas sensing. Therefore, total time of the ERS tasks execution becomes more definite and is easier to predict.

Let us consider the experiment in the ERS tasks execution time analysis under the condition of several spacecrafts leaving the swarm. Let us use the example of sensing of four observation areas 
Table 1: Comparison of sensing time.

\begin{tabular}{|c|c|c|c|c|c|c|c|}
\hline \multicolumn{2}{|c|}{ Number of observation areas } & \multirow{2}{*}{$\frac{1}{42.8}$} & \multirow{2}{*}{$\frac{2}{57.4}$} & \multirow{2}{*}{$\frac{3}{66.6}$} & \multirow{2}{*}{$\begin{array}{c}4 \\
103\end{array}$} & \multirow{2}{*}{$\frac{5}{103.8}$} & \multirow{2}{*}{$\frac{6}{130.8}$} \\
\hline & & & & & & & \\
\hline & & 43.6 & 57.8 & 59.4 & 86.8 & 90.6 & 117.2 \\
\hline & & 2. & 2. & 1. & 8. & 18. & 25.4 \\
\hline & Real-time scheduling & 3.07 & 1.72 & 2.5 & 3.76 & 3.61 & 13.4 \\
\hline
\end{tabular}

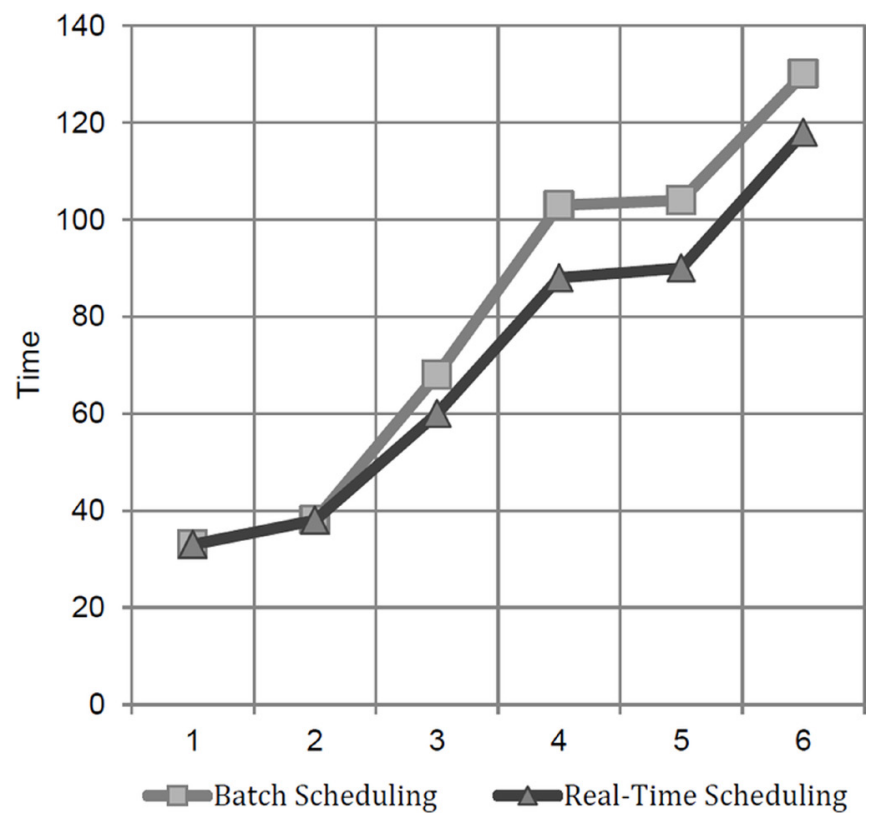

Figure 4: The average sensing time chart.

that are evenly distributed along the Earth's equator. The number of areas and their locations remain the same throughout all the experimental series. Several spacecrafts are dynamically eliminated from the swarm. The number of eliminated spacecrafts increases in each next series of the experiment. The selection criterion of the eliminated satellite is its mandatory participation in the sensing process of one of the observation areas. For each experiment, the result will be total time of the observation areas sensing by the resources of the rest of the satellite swarm.

Table 2 shows the time values (in terms of simulated time) spent on the observation areas sensing and that are obtained in the course of the experiment. A graphical representation of the experimental results is shown in Fig. 5.

Comparison of the sensing duration in an experimental series that has a different number of eliminated spacecrafts has shown that with each SC elimination, its tasks are redistributed among other spacecrafts of the swarm. Moreover, the sensing task solution takes more time with the new conditions, as the new executors watch the observation area in less time than the eliminated ones. 
Table 2: Observation areas sensing time, under the condition of spacecraft elimination.

\begin{tabular}{lrrrrrrrr}
\hline & \multicolumn{7}{c}{$\begin{array}{c}\text { Observation areas sensing rime, in simulated time terms, } \\
\text { considering eliminated spacecraft }\end{array}$} \\
\cline { 2 - 9 } $\begin{array}{l}\text { Series } \\
\text { no. }\end{array}$ & \multicolumn{1}{c}{0} & \multicolumn{1}{c}{1} & \multicolumn{1}{c}{2} & 3 & 4 & 5 & 6 & 7 \\
\hline 1 & 72 & 114 & 110 & 142 & 130 & 165 & 197 & 364 \\
2 & 80 & 95 & 118 & 126 & 169 & 174 & 238 & 411 \\
3 & 69 & 92 & 129 & 138 & 134 & 168 & 226 & 376 \\
4 & 70 & 101 & 115 & 131 & 126 & 189 & 245 & 382 \\
5 & 81 & 117 & 134 & 122 & 151 & 175 & 223 & 447 \\
$\bar{x}$ & 74.4 & 103.8 & 121.2 & 131.8 & 142 & 174.2 & 225.8 & 396 \\
\hline
\end{tabular}

\section{Time}

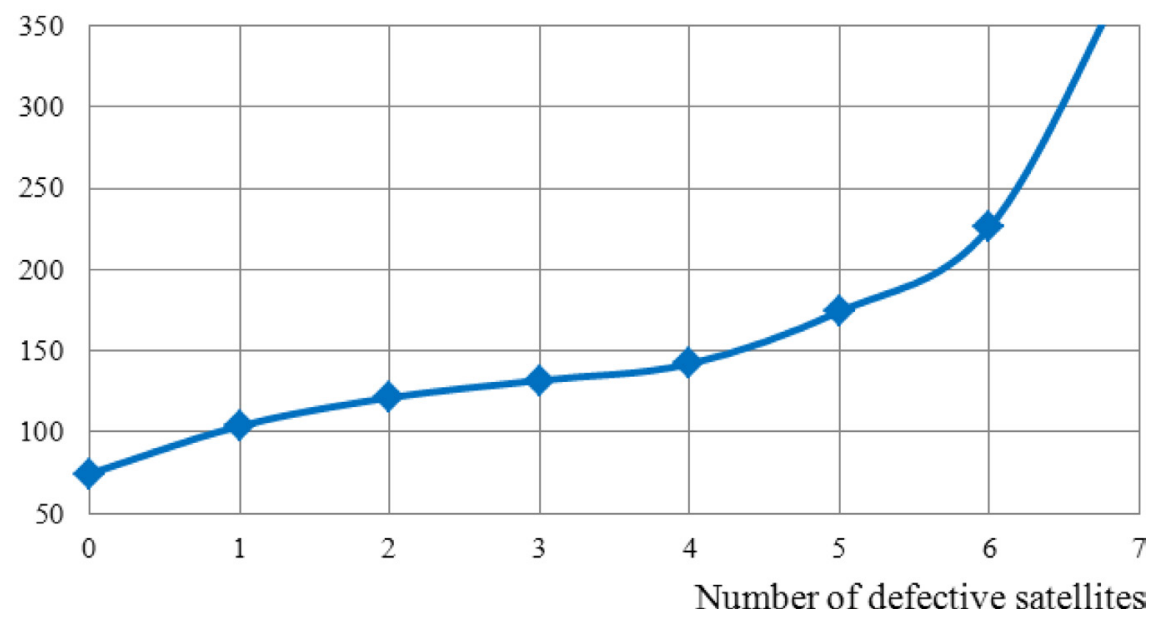

Figure 5: Average sensing time under the condition of SC elimination.

\section{CONCLUSION}

The experiments conducted have shown the potential of the suggested approach to the swarm of spacecraft management in terms of complex dynamic environments. The solutions must not only be optimal in a global sense, but also appropriate and fast in the current situation. Preliminary results show that adaptive pattern recognition and scheduling generate results of higher quality faster and cheaper than in previous arrangements. In addition, the solution is scalable and reliable.

\section{ACKNOWLEDGMENTS}

This work was carried out in SEC 'Smart Solutions' Ltd with the financial support of the Ministry of Education and Science of the Russian Federation (Contract no. 14.576.21.0012, unique number RFMEFI57614X0012). P. Skobelev was the project manager and E. Simonova the senior analyst.

\section{REFERENCES}

[1] Tripp, H. \& Palmer, P., Stigmergy based behavioural coordination for satellite clusters, Acta Astronautica journal, 66, pp. 1052-1071, 2010. 
134 P. Skobelev et al., Int. J. of Design \& Nature and Ecodynamics. Vol. 11, No. 2 (2016)

[2] Iacopino, C., Palmer, P., Policella N., Donati A., Brewer A. How Ants Can Manage Your Satellites, Acta Futura, 9, pp. 57-70, 2014.

[3] Wooldridge, M., An Introduction to Multiagent Systems, John Wiley \& Sons: London, 2nd edition, 2009.

[4] Rzevski, G. \& Skobelev, P., Managing complexity, WIT Press: London-Boston, 1st edition, 2014.

[5] Sollogub, A., Intelligent System for Distributed Problem Solving in Cluster of Small Satellites for Earth Remote Sensing, Information and Control Systems, eds. P. Skobelev, E. Simonova, A. Tzarev., M. Stepanov, A. Zhilyaev, 1(62), pp. 16-26, 2013.

[6] Skobelev, P., Activity ontology for situational management of enterprises in real time, Ontology of designing, 1(3), pp. 6-38, 2012. 